\title{
Aspectos críticos do controle do dengue no Brasil
}

\author{
Critical aspects of dengue control in Brazil
}

\footnotetext{
1 Faculdade de Medicina Área de Medicina Social, Universidade de Brasília. Campus Universitário Darcy Ribeiro, Asa Norte Brasília, DF 70910-900, Brasil. pltauil@unb.br
}

\begin{abstract}
Dengue is now the main reemerging disease in the world. In the absence of an efficaci ous preventive vaccine and effective etiol ogic treatment and chemoprophylaxis, the only vulnerable link for reducing dengue transmission is the mosquito Aedes aegypti, its principal vector. There are many difficulties in combating this mosquito in large and medium-sized cities. The complexity of contemporary urban life generates factors that facilitate the mosquito's proliferation and constraints on the reduction of its infestation rates. The objectives of dengue control should be based on available scientific and technical knowledge. Thus, while it is not possible to avoid dengue in areas infested with A. aegypti, it is possible to prevent major epidemics by improving epidemi ol ogical surveillance, and it is both possible and feasible to reduce the disease's case fatality from the current 5 to $6 \%$ to some $1 \%$ in the severe forms. The elaboration and execution of strategic plans for the organization of medical care for suspected dengue cases have proven to be a highly useful instrument to reduce case fatality both in other countries and in some cities of Brazil.
\end{abstract}

Key words Vector Control; Disease Outbreak; Aedes; Dengue; Epidemi ologic Surveillance

Resumo O dengue é hoje a principal doença re-emergente no mundo. Na ausência de uma vacina preventiva eficaz, de tratamento etiológi co equimioprofilaxia efetivos, o único el o vulnerável para reduzir a sua transmissão é o mosquito Aedes aegypti, seu principal vetor. As dificuldades de combater este mosquito, em grandes e médias ci dades, são muitas. Há facilidades para sua prol iferação e li mitações para reduzir seus índices de infestação, geradas pela complexi dade da vi da urbana atual. Os objetivos do controle do dengue devem ser estabel eci dos com base nos conhecimentos ci entíficos e técnicos disponíveis. Assi m, não sendo possível evitar casos de dengue em áreas infestadas pelo A. aegypti, é possível preveni r epi demias de grandes dimensões por meio do aprimoramento da vigilância epidemiológica, eé possível e factível reduzir a letalidade da doença, dos níveis atuais de 5 a $6 \%$ para cerca de $1 \%$ das formas graves. A elaboração e execução de planos estratégi cos de organização da assi stência aos casos su speitos de dengue têm mostrado, tanto em outros países, como em algumas ci dades brasi leiras, ser um instrumento muito útil na redução da letalidade.

Palavras-chave Controle deVetores; Surto de Doenças; Aedes; Dengue; Vigilância Epi demiológica 
O controle de um agravo à saúde pode ter diferentes objetivos em função do grau de conhecimento científico que se tenha do agravo, dos recursos tecnológicos disponíveis e das condições sócio-econômicas e políticas existentes. Entre a erradicação, objetivo mais ambicioso, e a redução da mortalidade específica pelo agravo, objetivo mais restrito, passando pela sua eliminação, redução da sua incidência ou da sua gravidade, estabelecem-se os objetivos das atividades de controle específicas e determinam-se as medidas preventivas disponíveis e consistentes a serem utilizadas. Em publicação de 1998, as relações entre objetivos de controle e medidas preventivas foram discutidas e aprofundadas (Tauil, 1998).

No que se refere ao dengue, é preciso que se determine, diante dos conhecimentos científicos e tecnológicos disponíveis, quais são os objetivos das atividades de controle passíveis de ser alcançados, estabelecendo-se as medidas preventivas adequadas a estes objetivos.

$O$ dengue é hoje a arbovirose mais importante do mundo. Cerca de 2,5 bilhões de pessoas encontram-se sob risco de se infectarem, particularmente em países tropicais onde a temperatura e a umidade favorecem a proliferação do mosquito vetor. Entre as doenças reemergentes é a que se constitui em problema mais grave de saúde pública. São bem conhecidas sua etiologia e seus mecanismos de transmissão. O seu espectro clínico é muito amplo, variando de formas assintomáticas ou oligosintomáticas até formas graves e letais. As causas da ocorrência de formas graves ainda não estão plenamente estabelecidas, existindo algumas teorias explicativas relacionadas à maior virulência da cepa de vírus infectante, à seqüência de infecções pelos diferentes sorotipos do agente etiológico, a fatores individuais do hospedeiro e a uma combinação de todas as explicações anteriores. Por outro lado, apesar de muito pesquisada, ainda não está disponível uma vacina preventiva eficaz. Da mesma forma, não se pode contar ainda com uma terapêutica etiológica e uma quimioprofilaxia efetivas. No momento, o único elo vulnerável na cadeia de transmissão do dengue a uma medida preventiva é o vetor.

\section{Aspectos relativos ao combate vetorial}

O mosquito Aedes aegypti é a principal espécie responsável pela transmissão do dengue. É um mosquito doméstico, antropofílico, com atividade hematofágica diurna e utiliza-se preferencialmente de depósitos artificiais de água limpa para colocar os seus ovos. Estes têm uma alta capacidade de resistir à dessecação, mantendo-se viáveis na ausência de água por até 450 dias. O A. aegypti tem mostrado uma grande capaci dade de adaptação a diferentes situações ambientais consideradas desfavoráveis. Adultos já foram encontrados em altitudes elevadas e larvas em água poluída.

A re-emergência do dengue está diretamente relacionada à reinfestação do país pelo $A$. aegypti. Antes da epidemia de Boa Vista, Roraima, em 1981/ 1982, o último registro da ocorrência de dengue havia acontecido há quase sessenta anos, em 1923 (Pedro, 1923). É possível que a doença possa ter passado despercebida, mas o fato é que nesse período a luta contra o mosquito foi intensa, particularmente com a finalidade de eliminar a forma urbana da febre amarela, também transmitida por este inseto. Nas décadas de 1950 e 1960, o Brasil e mais 17 países das Américas conseguiram eliminá-lo de seus territórios. A estratégia utilizada foi a de uma campanha nacional, centralizada, verticalizada, com estruturação militar, onde a disciplina e a hierarquia eram características marcantes. Porém, a partir de uns poucos países que não obtiveram o mesmo êxito, o Brasil enfrentou centenas de re-infestações, as quais foram detectadas precocemente e eliminadas. Em 1976, foi detectada uma infestação que não pôde ser eliminada, disseminando-se para outros estados como o Rio Grande do Norte e o Rio de Janeiro. Daí, o A. aegypti re-infestou todas as Unidades da Federação e atual mente já foi detectado em quase 4 mil municípios.

A luta contra este mosquito apresenta muitos pontos críticos. Não se sabe qual o índice de infestação abaixo do qual a transmissão de dengue se interrompe (Gomes, 1998). Dois índices são os mais usados: o de infestação predial (percentual de prédios encontrados com recipientes contendo água e larvas em relação ao número total de prédios examinados) e o de Bretau (percentual de recipientes encontrados com larvas em relação ao número total de prédios examinados). Nenhum deles é suficientemente capaz de medir a intensidade de infestação (Gomes, 1998). No primeiro caso, um prédio pode ter um ou vários recipientes positivos para larvas e é considerado apenas como um prédio infestado. No caso do Índice de Bretau, não se diferencia o tipo de reservatório, contabilizando da mesma forma um tonel de água com larvas e um prato de xaxim com larvas, embora o número de larvas num tonel seja em geral, muitas vezes maior que no prato de xaxim. Na literatura, há referência de que com um índice de infestação predial menor que 1\% 
e um índice de Bretau abaixo de 5\% não haveria transmissão de dengue. Porém, Kuno relata que houve transmissão de dengue em Cingapura com índice de Bretau abaixo de 5\% (Kuno, 1995). Portanto, baixos índices de infestação pelo A. aegypti reduzem o risco de transmissão de dengue, porém não o eliminam. Há ainda a necessidade de manter esses índices baixos, o que exige uma vigilância entomológica permanente, atividade essa intensiva de mão-de-obra e que, com a redução da incidência da doença, perde, com o passar dos anos, o seu apelo político e, por conseqüência, recursos financeiros, materiais e humanos, em favor de outras prioridades em saúde.

Outro vetor transmissor de dengue no Sudeste Asiático, existente no Brasil desde 1986, é o Aedes al bopictus, até agora não encontrado naturalmente infectado no país. Possui uma valência ecológica bem mais ampla que o A. aegypti, sendo encontrado também em ambiente silvestre, não passível portanto de eliminação. É um vetor secundário, uma vez que não é muito doméstico e nem muito antropofílico. Assim, mesmo que o A. aegypti seja eliminado, ainda existe, mesmo que reduzido, o risco de transmissão de dengue pelo $\mathrm{A}$. al bopictus.

Por que o A. aegypti se multiplica e se dissemina tanto nos dias atuais? A sua proliferação nas Américas, e em particular no Brasil, tem múltiplos condicionantes. O fluxo rural-urbano intenso nos últimos trinta anos, resultou numa concentração populacional muito elevada em médias e grandes cidades. Mais de $80 \%$ da população brasileira vive hoje em área urbana. As cidades, pressionadas por essa demanda, não conseguiram oferecer condições satisfatórias de habitação e de saneamento básico a uma fração importante dos seus habitantes: em torno de $20 \%$ vivem em favelas, invasões, mocambos ou cortiços, onde, quando existem, o abastecimento de água e a coleta de dejetos, são irregulares. A necessidade de armazenar água para consumo em tonéis é um fator que favorece a proliferação do mosquito vetor. O privilegiamento, pelo processo industrial moderno, de embalagens descartáveis, contribui para a multiplicação dos mosquitos quando estas embalagens, de plástico, alumínio, vidro ou isopor, não são adequadamente recolhidas após sua utilização. O fantástico aumento da produção de veículos automotores contribui igualmente para a multiplicação do vetor, na medida em que aumenta o número de pneus usados dispostos inadequadamente no meio ambiente, comportando-se como recipientes prioritários para a postura de ovos pelos mosquitos, e permitindo o transporte passi- vo de ovos, larvas e insetos adultos facilitando a sua disseminação.

Além das facilidades de proliferação e disseminação do A. aegypti oferecidas pelas condições atuais de vida urbana, o combate ao mosquito também apresenta limitações. Do ponto de vista institucional, alguns aspectos críticos podem ser detectados. As atividades de vigilância sanitária em nível municipal carecem de legislação de apoio, e/ ou de práticas de fiscalização, para eliminarem os criadouros do mosquito em pontos considerados estratégicos. Estes são as borracharias, com pneus expostos às intempéries do tempo; os cemitérios, com seus múltiplos vasos acumulando água; os depósitos de ferro velho a céu aberto retendo água de chuva; os terrenos baldios não cuidados, com múltiplos recipientes retendo água e as caixas d'água domiciliares descobertas. Outra dificuldade atual relacionada ao poder público é a ampliação e regularização do abastecimento de água encanada e da coleta freqüente do lixo, com destinação adequada, particularmente nas periferias das cidades. O terceiro componente institucional crítico é a inspeção predial e eliminação ou tratamento de reservatórios potenciais ou atuais de larvas de mosquito, e aplicação de inseticida em locais com transmissão ativa da doença. As grandes e médias cidades possuem hoje áreas de difícil acesso aos domicílios pelos servidores públicos, por razões de segurança: tanto em bairros de população mais abastada, como naqueles mais pobres. As inspeções são feitas durante o dia e muitos prédios encontram-se fechados, em função das atividades laborais de seus ocupantes. Dessa forma, a inspeção fica quantitativamente prejudicada e muitos focos de mosquito não identificados e, portanto, não tratados. Como é uma atividade intensi va de mão-de-obra, a inspeção de domicílios para levantamento dos índices de infestação e eliminação de focos, exige contratação, treinamento e supervisão de pessoal de campo, em quantidade suficiente para dar cobertura abrangente dos domicílios. Em virtude de limitações legais para contratação de pessoal, muitos municípios têm utilizado a terceirização como meio de superar as dificuldades legais. Os contratos, em geral, são temporários e às vezes sem garantias trabalhistas. Ora, como os servidores não permanecem muito tempo em empregos tão instáveis, mesmo que sejam bem treinados, não chegam a adquirir experiência suficiente para um trabalho de boa qualidade. A supervisão, por outro lado, é quase inexistente. Finalmente, o quarto elemento institucional importante é aquele relativo à informação, educação e co- 
municação da população sobre a necessidade e as formas de reduzir os fatores domiciliares que favorecem a multiplicação dos mosquitos. A mobilização comunitária para a adoção de práticas de redução dos vetores é de fundamental importância. Muitas vezes, a população tem a informação correta, porém suas práticas não são coerentes com o conhecimento do problema. A abordagem do assunto pelos meios de comunicação e pelas escolas, deve buscar justamente a mudança das práticas habituais facilitadoras da proliferação do A. aegypti.

Em função da situação político-institucional do Brasil, particularmente do setor saúde, não se admite mais uma estratégia de combate ao mosquito nos moldes da realizada no passado, por meio de uma campanha centralizada, verticalizada e hierarquizada. Porém, não há experiência no mundo de eliminação de um vetor de doença realizada de forma descentralizada, com direção única em cada nível de governo, a exemplo do preconizado pelo Sistema Ú nico de Saúde brasileiro. Muito ainda precisa ser feito para a aquisição de uma estratégia efetiva de combate ao vetor do dengue de forma descentralizada. O Brasil eliminou o Anopheles gambiae do Nordeste, no início da década de 1940, com uma campanha centralizada. O êxito dessa campanha foi o principal motivo para a realização da chamada campanha de erradicação continental do A. aegypti, na década de 1950, nos mesmos moldes da campanha do gambiae (Franco, 1969).

O combate ao principal vetor do dengue encontra atualmente um novo ponto crítico. Tratase do problema da resistência crescente dos insetos aos larvicidas e adulticidas de uso habitual nas atividades de controle. O mosquito já apresentava, há muitos anos, resistência aos inseticidas organoclorados. Muitos pesquisadores estão relatando resistência das larvas e dos adultos aos inseticidas organofosforados e dos adultos aos piretróides. Assim, o monitoramento da resistência dos mosquitos deve ser permanente, bem como a pesquisa de novos produtos inseticidas, eficazes e ecologicamente seguros.

As dificuldades de combater o A. aegypti são muito grandes nos dias de hoje. Alguns exemplos históricos recentes precisam ser lembrados, entre eles o de Cuba. Após enfrentar heroicamente a primeira epidemia de dengue hemorrágico nas Américas, em 1981, Cuba empreendeu uma luta nacional para erradicar o vetor de seu território, em moldes campanhistas. Conseguiu reduzir drasticamente os índices de infestação. Porém, a partir de um resíduo mínimo de infestação, o A. aegypti novamente reapareceu transmitindo dengue, inclu- sive recentemente, em fins de 2001 e início de 2002. É preciso levar-se em conta que Cuba é uma ilha, com cerca de 12 milhões de habitantes, e que desenvolveu uma campanha extremamente organizada, com participação intensa da população e forte apoio político. Outro exemplo é Cingapura, modelo internacional de combate ao vetor do dengue, que ainda apresenta um resíduo de infestação, insuficiente para impedir transmissão esporádica da doença. Todos os 18 países das Américas que eliminaram o A. aegypti dos seus territórios no passado, estão todos novamente re-infestados.

\section{Aspectos relativos ao controle da mortalidade}

Diante de tantas dificuldades para combater o mosquito e reduzir a transmissão do dengue, outros aspectos do seu controle precisam ser aprimorados. O primeiro deles refere-se à redução da mortalidade específica. É possível e factível reduzir a letalidade por dengue hemorrágico a valores em torno de $1 \%$. Nas epidemias de Cuba e da Venezuela, esses valores foram alcançados. No Brasil, a letalidade ainda está muito alta. Segundo dados da Fundação Nacional de Saúde, do Ministério da Saúde, em 2001, houve o registro de 675 casos de forma hemorrágica do dengue e 29 óbitos, um coeficiente de letalidade de 4,3\%. Neste início de 2002, principalmente na cidade do Rio de Janeiro, o número de óbitos aumentou muito, em função da circulação do sorotipo DEN-3. Ainda não se tem os dados consolidados, mas os disponíveis mostram uma letalidade acima dos 5\%, com 29 óbitos entre 460 casos de dengue hemorrágico, até o dia 4 de abril. $O$ aspecto crítico nessa questão diz respeito exclusivamente ao setor saúde. É preciso que cada cidade disponha de um plano estratégico de atendimento aos pacientes suspeitos de dengue, facilitando seu acesso precoce aos serviços de saúde. Estes devem contar com pessoal treinado nos procedimentos para classificar os casos, e nas condutas a serem tomadas segundo sua classificação. Como estas condutas variam desde o acompanhamento do doente em casa até a internação em unidades de terapia intensiva, é preciso organizar os serviços de referência dos doentes, reservar leitos hospitalares, manter os insumos necessários e pessoal capacitado para o atendimento nos diferentes níveis de complexidade da assistência médica. Algumas cidades brasileiras elaboraram e puseram em prática este plano e tiveram oportunidade de colher bons resultados, reduzindo substancialmente a leta- 
lidade por dengue hemorrágico. Ao lado dessas medidas, é preciso manter a população informada da possibilidade de formas graves e até letais da doença, estimulando a busca precoce de assistência médica.

\section{A prevenção de grandes epidemias}

Além de reduzir a mortalidade específica por dengue, é possível reduzir as dimensões das epidemias que têm, no Brasil, se tornado cada vez maiores e mais graves? A detecção precoce e a investigação de surtos de doença febril, sem confirmação diagnóstica, em área infestada pelo A. aegypti, seguida de medidas de controle localizadas, havendo a confirmação de dengue, é a forma mais eficaz de prevenir epidemias de grandes dimensões. $\mathrm{O}$ aspecto crítico aqui é a vigilância epidemiológica nos municípios. Essa atividade exige uma co-responsabilidade de todos os profissionais de saúde, tanto na área de assistência médica como na de saúde pública. Exige a instalação e funcionamento de laboratórios de apoio diagnóstico e monitoração dos sorotipos circulantes. Exige uma resposta rápida de combate ao vetor infectado, que se torna mais efetiva na medida em que a área de atuação é mais restrita. A vigilância epidemiológica é consi derada muitas vezes uma atividade apenas burocrática e não desperta o interesse, principalmente dos médicos dos serviços de saúde. Porém, a informação é o ponto de partida para desencadear ações de controle. A capacidade dos serviços de saúde de responder, com ações efetivas de controle, à notificação de transmissão de dengue localizada numa área geográfica restrita, é a forma possível de prevenir epidemias de grandes dimensões. Quando a doença já está ocorrendo simultaneamente em diferentes bairros da cidade, toda a atividade de combate ao vetor torna-se mais difícil, quando não se transforma em uma verdadeira guerra, em se tratando de uma cidade de grande ou médio porte. Faz parte ainda da vigilân-

\section{Referências}

FRANCO, O., 1969. História da Febre Amarela no Brasil. Rio de Janeiro: Superintendência de Campanhas de Saúde Pública, Ministério da Saúde.

GOMES, A. C., 1998. Medidas dos níveis de infestação urbana para Aedes (Stegomyia) aegypti e Aedes (Stegomyia) albopictus em programa de vigilância entomológica. Informativo Epidemiológi co do SUS, 7:49-57.

KUNO, G., 1995. Review of the factors modulating denguetransmission. Epidemiologic Review, 17:321-335. cia epidemiológica, manter a população de uma área infestada por A. aegypti informada da doença, de suas características clínicas e da necessi dade de buscar assistência médica precocemente se perceber os sintomas da doença. Infelizmente, é praticamente impossível, impedir a entrada de pessoas infectadas, em fase de transmissibilidade da doença, em áreas com a presença do vetor. Os meios atuais de transporte são muito rápidos e freqüentes, e podem deslocar indivíduos portadores do vírus de locais distantes, rapidamente. A identificação desses indivíduos para a tomada de medidas sanitárias adequadas, é totalmente inviável pela dificuldade de diagnóstico rápido, pelo número elevado de assintomáticos ou oligossintomáticos e pela intensidade do tráfego de passageiros, quer por via aérea, quer por via terrestre.

\section{Comentários finais}

Considerando os conhecimentos científicos e recursos tecnológicos atualmente disponíveis em relação ao dengue, os objetivos do controle desta doença devem estar bem claros. É possível reduzir os atuais coeficientes de letalidade para valores em torno de $1 \%$, organizando o sistema de assistência médica aos casos suspeitos. É possível reduzir as dimensões das epidemias, aprimorando o sistema de vigilância epidemiológica, detectando mais precocemente os surtos da doença e respondendo mais efetivamente no combate ao vetor infectado, quando presente apenas em áreas restritas das grandes e médias cidades brasileiras. Torna-se muito difícil, na presença do A. aegypti, mesmo em níveis baixos de infestação, evitar-se casos de dengue, pois a entrada do vírus, por meio de portadores, numa área infestada é praticamente impossível. Realisticamente, a eliminação desse vetor das grandes e médias cidades parece inexeqüível nos dias de hoje, considerando toda a complexidade da vida urbana.

PEDRO, A. O., 1923. Dengue em Nicteroy. Brasil-Mé dico, 1:173-177.

TAUIL, P. L., 1998. Controle de agravos à saúde: Consistência entre objetivos e medidas preventivas. Informativo Epidemiológico do SUS, 7:55-58.

Recebido em 8 de abril de 2002

Aprovado em 10 de abril de 2002 